Conclusion: The discrimination function is a proposed screening algorithm for pts with SSc-ILD who could develop progression disease. Usage of discrimination function revealed that $84 \%$ pts with ILD-SSc have got poor prognosis and $9 \%$ of them have got fatal outcome. Usage the equation of prognosis is very useful to choice of treatment and observation pts SSc-ILD.

Disclosure of Interests: None declared.

DOI: 10.1136/annrheumdis-2021-eular.3589

\section{AB0454 SPECIFICITY OF INTERSTITIAL LUNG DISEASE IN PATIENTS WITH SYSTEMIC SCLEROSIS POSITIVE FOR A-TOPO-1 AND A-RNP}

R. Shayakhmetova ${ }^{1}$, L. P. Ananyeva ${ }^{1}$, O. Koneva ${ }^{1}$, M. Starovoytova ${ }^{1}$,

O. Desinova', O. Ovsyannikova ${ }^{1}$, L. Garzanova', A. Khelkovskaia-Sergeeva ${ }^{1}$,

S. Glukhova' ${ }^{1}$ V.A. Nasonova Research Institute of Rheumatology; V.A.

Nasonova Research Institute of Rheumatology, Moscow, Russian Federation

Background: The classification criteria of SSC (EULAR/ACR 2013) compared to the criteria of 1980 showed high sensitivity and allow to detect the full spectrum of systemic sclerosis (SSc). The use of the new criteria led to the fact that some of the patients who were diagnosed with MCTD began to meet the criteria of SSc. Since these patients have hyperproduction of antibodies to RNP, but no other SSc-specific autoantibodies, we believe that they represent a special clinical and immunological phenotype of SSc. Lung involvement in SSc impaires the quality of life and the prognosis, so the study of interstitial lung disease(ILD) in different phenotypes is relevant.

Objectives: To compare the main manifestations of ILD in two groups of patients who meet the classification criteria of SSc 2013 - positive for a-Topo-1 and for a-RNP.

Methods: The study included 100 patients. The first group - 50 patients positive for a-Topo-1 (45 women and 5 men, mean age $55 \pm 10$ years, duration of the disease $10.2 \pm 8$ years, subset of the disease: diffuse $52 \%$, limited $48 \%$ ) and the second group - 50 patients positive for a-RNP ( 43 women and 7 men, mean age $44.5 \pm 14$ years, duration of the disease $11.7 \pm 8$ years; form of the disease: diffuse $14 \%$, limited 86 ). The diagnosis of ILD was established by HRCT.

Results: In patients of group I ILD was detected in $94 \%$ of cases, in group II - $68 \%(p<0.05)$. In both groups a decrease in functional lung tests was noted with a high frequency, but a severe decrease in the lung diffusion capacity was significantly more often observed in group I.

Table 1. Data of laboratory and instrumental parameters

\begin{tabular}{lccc}
\hline & a-Topo-1- positive & a-RNP-positive & \\
Parameters & $\mathrm{n}(\%)$ & $\mathrm{n}(\%)$ & $\mathrm{p}$ \\
\hline Decrease of DLCO $<80 \%$ & $49(98)$ & $43(86)$ & $\mathrm{p}<0,05$ \\
DLCO $<55 \%$, & $33(66)$ & $16(32)$ & $\mathrm{p}<0,05$ \\
Decrease of FVC $<80 \%$ & $19(38)$ & $10(20)$ & $\mathrm{p}<0,05$ \\
FVC $<70 \%$ & $13(26)$ & $7(14)$ & $\mathrm{NS}$ \\
Ground glass opacity & $25(50)$ & $26(52)$ & $\mathrm{NS}$ \\
Honeycombing & $17(34)$ & $8(16)$ & $\mathrm{p}<0,05$ \\
SPAP $>40 \mathrm{~mm} . \mathrm{Hg}$, & $6(12)$ & $10(20)$ & $\mathrm{NS}$ \\
including PAH & $2(4)$ & $3(4,6)$ & $\mathrm{NS}$ \\
ANA $>1: 640$ & $37(74)$ & $45(90)$ & $\mathrm{p}<0,05$ \\
\hline
\end{tabular}

* ANA - antinuclear antibodies, PAH - pulmonary arterial hypertension, SPAP - systolic pulmonary artery pressure ${ }^{* *}$ confirmed by the data of catheterization of the right heart

Typical changes according to HRCT were detected in both groups, but fibrotic changes dominates in group I. There were no significant differences in the frequency of occurrence of PAH. Patients of group II had high level of ANA more often.

Conclusion: In both groups, a high frequency and similar manifestations of ILD were noted. In patients with SSc positive for anti-U1RNP, despite the predominance of limited skin lesions (in $86 \%$ of patients), a high incidence of ILD was detected, while the frequency of PAH was comparable to SSc in general. Compared with a-Topo-1 positive patients, ILD was significantly less frequent in anti-U1RNP positive patients and was accompanied with a smaller reduction of DLCO and/or FVC and a lower frequency of severe lung fibrosis. These data confirm the similarity of lung involvement in compared phenotypes of SSc and indicate the importance for screening for ILD in patients with hyperproduction of a-RNP, especially at an early stage of the disease.

REFERENCES:

[1] Van den Hoogen F, Khanna D, Fransen J, et al. 2013 classification criteria for systemic sclerosis: an American College of Rheumatology/European League Against Rheumatism collaborative initiative. Ann Rheum Dis 2013;72:1747-55

Disclosure of Interests: None declared.

DOI: 10.1136/annrheumdis-2021-eular.3591

\section{AB0455 \\ MODIFICATION OF PROGNOSIS INTERVALS WAS DERIVED BY THE DISCRIMINATION FUNCTION FOR SSC-ILD PATIENTS}

O. Ovsyannikova ${ }^{1}$, L. P. Ananyeva ${ }^{1}$, L. Garzanova ${ }^{1}$, O. Koneva ${ }^{2}$, S. Glukhova ${ }^{2}$, M. Starovoytova ${ }^{1}$, O. Desinova ${ }^{1}$, R. Shayakhmetova ${ }^{1} .{ }^{1}$ VA Nasonova Research Institute of Rheumatology, Laboratory of Microcirculation and Inflammation, Moscow, Russian Federation; ${ }^{2}$ VA Nasonova Research Institute of Rheumatology, Educational and Methodical Department, Moscow, Russian Federation

Background: Systemic sclerosis (SSc) is a multisystem autoimmune disease Pulmonary alterations are the major cause of mortality in SSc. SSc-ILD has heterogeneous disease progression: many patients will have a chronic, indolent course while others may develop progressive, life-threatening disease.

Objectives: To assess prognosis intervals for pts with SSc-ILD.

Methods: It was a longitudinal study involving 140 pts with SSc-ILD. The mean age was $46.7 \pm 13.9$ years, females $82 \%$, SSc duration $6.2 \pm 5.8$ years, diffuse subset - $54 \%$. The mean duration of follow up was $73,2 \pm 27,8$ months. Clinical, laboratory, and immunologic data were collected on all patients.

Based on analysis of discrimination function the equation of prognosis was developed. The equation is $3.14 \mathrm{GGO}$ (ground glass opacities) $+0,7$ index EScSG - 1,326 FVC - 0,1 the maximum daily dose of glucocorticoids + 0,136 Gamma globulins $+1.066 \mathrm{CYP}-1.075 \mathrm{DLCO} \leq 5,8$. Value of equation of prognosis to 5.8 corresponds to stabilization or good prognosis and value after 5.8 corresponds to poor prognosis. The equation of prognosis had $86 \%$ sensitivity and $56 \%$ specificity. The cut-off point 4.7 was represented $65 \%$ sensitivity and $67 \%$ specificity. The prognosis intervals were following: value of equation to 4.7 corresponds to good prognosis; value from 4.8 to 5.8 corresponds to stabilization and value after 5.8 corresponds to poor prognosis.

Results: Pts were distributed into groups depending on results of discrimination function: 14 pts (10\%) - zone of good prognosis; 8 pts $(6 \%)$ - zone of stabilization and $118(84 \%)$ - zone of poor prognosis. We have decided to join pts from zone good prognosis and zone stabilization into united group (U-group) - 22 pts $(16 \%)$

Table 1. Comparative characteristics of the groups

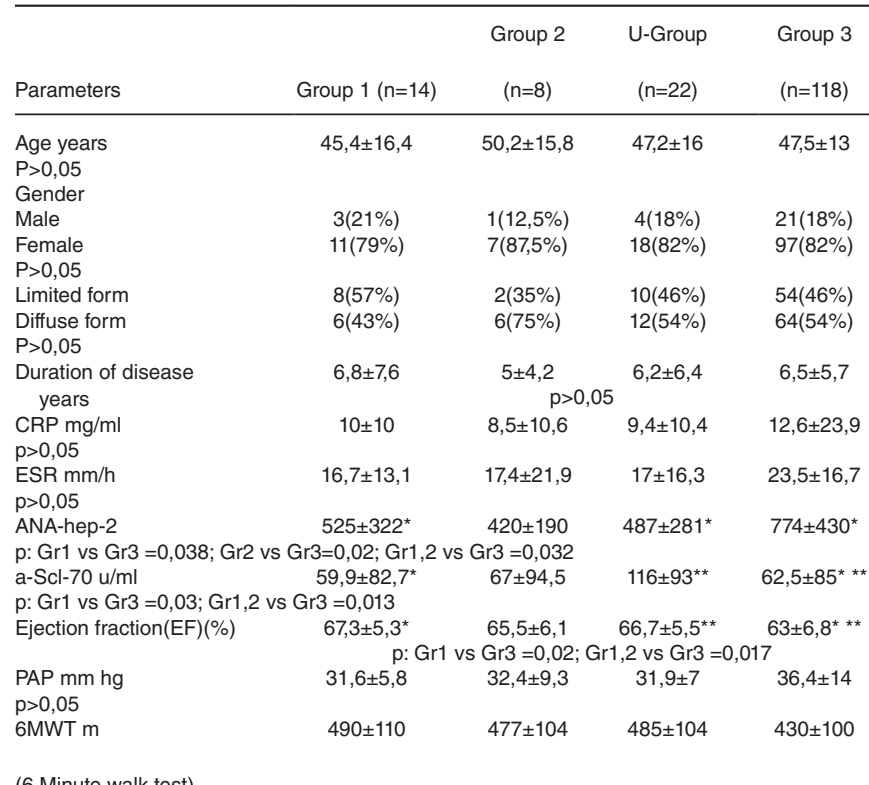

$p>0,05$

The age, SSc duration, gender proportion and SSc forms, were similar in the all groups $(p>0,05)$. In the study mean dates of ANF-hep-2, a-Scl-70 were significantly lower and EF was significantly higher in groups 1 and U-group than in group 3. In groups 1,2 and U-group the mean levels of ESR, CRP PAP were lower and value of $6 \mathrm{MWT}$ were higher than mean levels in group 3 accordingly. So separately group 2 didn't have significant differences with group 1 and 3 .

Conclusion: In the study we didn't find any correlation between meanings of group 2 and groups 1,3 but the meanings of group 1 and U-group have had the 
same correlation. It would be rational to highlight only one zone of prognosis before and after 5,8 .

Disclosure of Interests: None declared.

DOI: 10.1136/annrheumdis-2021-eular.3627

\section{AB0456 HYDROXYCHLOROQUINE MIGHT REDUCE MORTALITY IN PATIENTS WITH SYSTEMIC SCLEROSIS}

M. Ozmen ${ }^{1}$, E. Otman Akat ${ }^{1}$, S. Gucenmez ${ }^{1}$, G. Kabadayi ${ }^{1}$, E. Durak Ediboglu ${ }^{1}$, G. Alp ${ }^{1}$, H. Cinakli ${ }^{1}$, E. Erpek ${ }^{1}$, I. Kurut Aysin ${ }^{1}$, O. Bayindir ${ }^{1}$, D. Solmaz ${ }^{1}$ S. Akar. ${ }^{1}{ }^{1}$ Izmir Katip Celebi University Faculty of Medicine, Rheumatology, Izmir, Turkey

Background: Systemic sclerosis (SSc) is a devastating disease that has a profound impact on life expectancy, reflected by a standardised mortality ratio of 3,5. There is still limited data regarding the predictive factors for mortality in patients with SSc. Determining those factors could guide in disease management and follow up. ${ }^{1}$

Objectives: We aimed to identify the predictive factors for death in SSc.

Methods: Patients followed in a tertiary rheumatology clinic in the last 5 years were included in this retrospective study. All of the patients met the ACR / EULAR SSc 2013 criteria. Medical records of the patients were reviewed. Follow up time was defined as the time period from the first admission of the patient to our rheumatology clinic until the date of death or the date on which the study was performed. Candidate predictive factors for mortality were tested by Kaplan-Meier (with Log rank) and Cox-regression analyses.

Results: In total 146 patients (mean age $55.6 \pm 12.3$ years, female $89.7 \%$, diffuse cutaneous type SSc $45.2 \%$ ) were included in the study (Table 1). The mean age at diagnosis of study group was $48 \pm 13.7$ years. The median duration of follow up was 71 (6-228) months. Fourteen (10\%) patients died during follow-up. The causes of death were: pulmonary (7), renal (2) and cardiac diseases (1), infection (3) and cancer (1).

Univariate analysis revealed that age at diagnosis $(p=0.028)$, SSc subtype $(p=0.035)$, the presence of interstitial lung disease $(p=0.002)$, oesophageal involvement (on computed tomography) $(p=0.030)$, pulmonary artery systolic pressure of $\geq 35 \mathrm{mmHg}$ (measured by transthoracic echocardiography) $(p=0.004)$, glucocorticoid $(p=0.029)$, hydroxychloroquine $(p=0.002)$ and cyclophosphamide $(\mathrm{p}=0.006)$ usage at any time were associated with mortality (Figure 1). Multivariate analyses model formed with age at diagnosis (B: 0.055 , $95 \% \mathrm{Cl}, 1.005-1.112 ; \mathrm{p}=0.033$ ), SSc subtype (B: $0.963,95 \% \mathrm{Cl} 0.541-12.684$; $\mathrm{p}=0.231$ ), glucocorticoid ( $\mathrm{B}: 1.396,95 \% \mathrm{Cl}, 0.487-33.507 ; \mathrm{p}=0.196)$ and hydroxychloroquine usage ( $\mathrm{B}:-1.50,95 \% \mathrm{Cl}, 0.061-0.816 ; \mathrm{p}=0.023$ ) showed that age at diagnosis and hydroxychloroquine usage were independent predictive factors for mortality in patients with SSc.

Conclusion: The results of the study revealed for the first time that apart from the age at diagnosis hydroxychloroquine might reduce mortality in patients with SSc. Further studies are needed to prove of this information.

REFERENCES:

[1] Elhai $M$, et al. Ann Rheum Dis 2017;0:1-9. doi:10.1136/ annrheumdis-2017-211448

Table 1. The demographic and clinical features in patients with systemic sclerosis.

\begin{tabular}{lc}
\hline Characteristic & Baseline \\
\hline Age at diagnosis & $48 \pm 13.7$ \\
Female sex, $\mathbf{n}(\%)$ & $131(89.7)$ \\
Duration of follow-up, months ${ }^{* *}$ & $71(6-228)$ \\
Disease subtype, $\mathbf{n}(\%)$ & \\
Diffuse / Limited & $66(45.2) / 80(54.8)$ \\
Autoantibodies, $\mathbf{n}(\%)$ & \\
Anti-Scl70 antibody & $50 / 143(35.0)$ \\
Anti-Centromere antibody & $62 / 143(43.4)$ \\
Immunsuppresive medication, ever, $\mathbf{n}(\%)$ & \\
Hydroxychloroquine & $91 / 143(63.6)$ \\
Mycophenolate mofetil & $18 / 145(12.4)$ \\
Azathioprine & $47 / 145(32.4)$ \\
Cyclophosphamide & $24 / 145(16.6)$ \\
Glucocorticoid & $80 / 140(57.1)$ \\
Others, $\mathbf{n}$ (\%) & \\
ILD & $68 / 130(52.3)$ \\
Pericardial effusion, ever & $26 / 133(19.5)$ \\
Esophageal dilation (detected by CT) & $51 / 128(39.8)$ \\
sPAP $\geq 35 m m H g$, ever (measured by ECHO) & $46 / 142(32.4)$
\end{tabular}

"Parameter presented as mean \pm SD"Parameter presented as median (min-max)CT, computed tomography; ECHO, echocardiogram; ILD, interstitial lung disease; SPAP, systolic pulmonary artery pressure

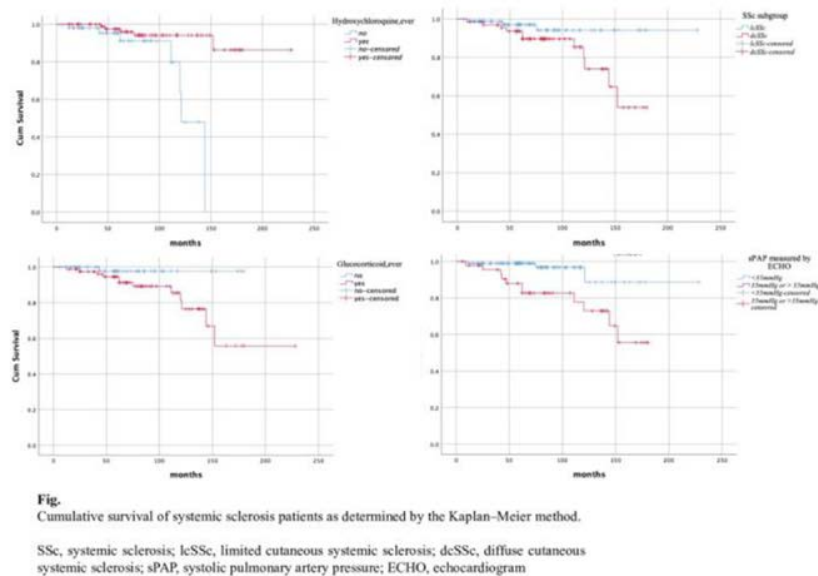

Figure 1.

Disclosure of Interests: None declared.

DOI: 10.1136/annrheumdis-2021-eular.3685

\section{AB0457 HIPPOCAMPAL SUBFIELDS VOLUMES REDUCTION IN PATIENTS WITH SYSTEMIC SCLEROSIS: A LONGITUDINAL MAGNETIC RESONANCE IMAGING (MRI) VOLUMETRIC STUDY}

D. Pereira ${ }^{1}$, M. Freschi ${ }^{1}$, R. Frittoli ${ }^{1}$, A. C. Londe ${ }^{1}$, T. Amaral $^{2}$, S. Dertkigil ${ }^{3}$, A. P. Del Rio ${ }^{4}$, F. Cendes ${ }^{5}$, L. Rittner ${ }^{6}$, S. Appenzeller ${ }^{4} .{ }^{1}$ UNICAMP, Pathophysiology Graduate Program, Campinas, Brazil; ${ }^{2}$ UNICAMP, Medicine, Campinas, Brazil; ${ }^{3}$ UNICAMP, Radiology, Campinas, Brazil; ${ }^{4}$ UNICAMP, Orthopedics, Rheumatology and Traumatology, Campinas, Brazil; ${ }^{5}$ UNICAMP, Neurology, Campinas, Brazil; ${ }^{6}$ UNICAMP, Electrical Engeneering, Campinas, Brazil

Background: Systemic sclerosis (SSc) is a multisystem disease characterized by functional and structural abnormalities of small blood vessels, fibrosis of the skin and internal organs. In addition, volume reduction and shape abnormality of the hippocampus have been demonstrated in rheumatic and neurodegenerative diseases. However, the neuroanatomy of the hippocampus is complex and is not a uniform structure, consisting of subfields with distinct morphology: cornu ammonis (CA) subfields CA1-4, dentate gyrus (DG), fimbria, and adjacent subiculum and presubiculum [1].

Objectives: To investigate the hippocampal subfields volumes reduction in SSc patients using MRI

Methods: In this study we included 37 SSc patients (33 women, mean age of $53.46, S D \pm 12.29$; range $30-78$ ) and 37 healthy controls $(\mathrm{HC})$ ( 31 women, mean age of $48.41, S D \pm 12.20$; range $29-80)$. Cognitive evaluation was performed using the Montreal Cognitive Assessment (MoCA), mood disorders were determined through Beck's Depression (BDI) and Beck's Anxiety Inventories (BAI). SSc patients were further assessed for clinical and laboratory SSc manifestations, disease activity (Valentini Activity Index), severity activity (Medsger Severity Index). MRI protocol consisted of: Sagittal T1-weighted images performed with a Philips 3.0T MRI scanner. MRI brain structure volumetric was done through volBrain [2]. After 48 months MRI acquisition were repeated in 26 SSc patients and 12 healthy volunteers. Statistics was performed according nature of the variable.

Results: We observed a significant reduction in hippocampal subfields volumes in SSc patients when compared to controls: Total hippocampi (SSc: mean volume = $4.78 \mathrm{~cm}^{3} ; \mathrm{SD}=0.38 ; \mathrm{HC}$ : mean volume $=5.01 \mathrm{~cm}^{3} ; \mathrm{SD}=0.38 ; \mathrm{p}=0.033$ ). Reduction in volume of the total hippocampi was associated with Raynaud's phenomenon $(p=0.006)$. A longitudinal study showed a reduction in volume of the hippocampus subfields volumes when compared to patient's baseline: Total hippocampi (mean initial volume $=4.78 \mathrm{~cm}^{3} ;$ mean follow-up volume $=4.50 \mathrm{~cm}^{3}, \mathrm{p}=0.027$ ); Total CA1 (mean initial volume $=1.59 \mathrm{~cm}^{3}$; mean follow-up volume $=1.58 \mathrm{~cm}^{3}, p<0.0001$ ); Reduction in volume of the total hippocampi was associated with presence of current use of prednisone $(p=0.008)$. Reduction in left CA1 left associated with current use of prednisone $(p=0.014)$. Reduction in total subiculum was associated with presence of calcinosis $(p=0.023)$. No significant changes were observed in hippocampal subfields volumes in controls over the follow-up period.

Conclusion: This study provides evidence of hippocampus subfields volumes reductions in SSc patients when compared to controls and was associated with 